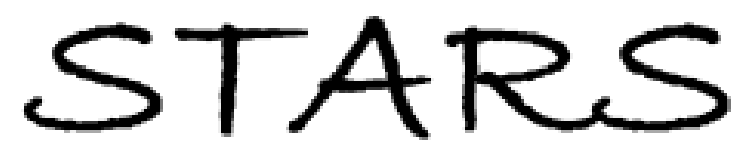

University of Central Florida

STARS

$1-1-2008$

\title{
Liquid crystal display using combined fringe and in-plane electric fields
}

Ji Woong Park

Young Joo Ahn

Jun Ho Jung

Seung Hee Lee

Ruibo Lu

University of Central Florida

See next page for additional authors

Find similar works at: https://stars.library.ucf.edu/facultybib2000

University of Central Florida Libraries http://library.ucf.edu

This Article is brought to you for free and open access by the Faculty Bibliography at STARS. It has been accepted for inclusion in Faculty Bibliography 2000s by an authorized administrator of STARS. For more information, please contactSTARS@ucf.edu.

\section{Recommended Citation}

Park, Ji Woong; Ahn, Young Joo; Jung, Jun Ho; Lee, Seung Hee; Lu, Ruibo; Kim, Hyang Yul; and Wu, ShinTson, "Liquid crystal display using combined fringe and in-plane electric fields" (2008). Faculty Bibliography 2000s. 818.

https://stars.library.ucf.edu/facultybib2000/818

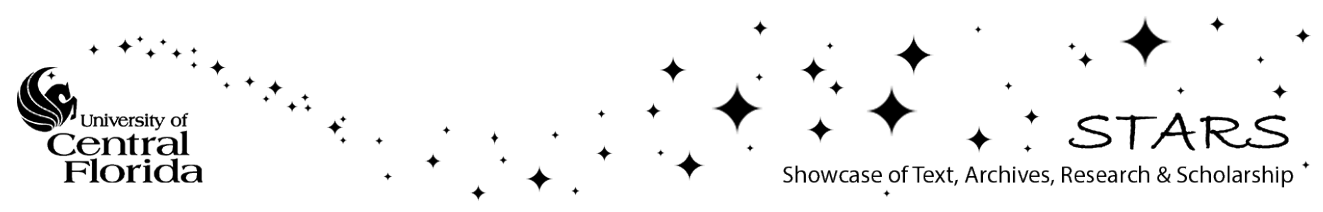


Authors

Ji Woong Park, Young Joo Ahn, Jun Ho Jung, Seung Hee Lee, Ruibo Lu, Hyang Yul Kim, and Shin-Tson Wu 


\section{Liquid crystal display using combined fringe and in-plane electric fields}

Cite as: Appl. Phys. Lett. 93, 081103 (2008); https://doi.org/10.1063/1.2973152

Submitted: 04 April 2008 . Accepted: 30 July 2008 . Published Online: 25 August 2008

Ji Woong Park, Young Joo Ahn, Jun Ho Jung, Seung Hee Lee, Ruibo Lu, Hyang Yul Kim, and Shin-Tson Wu

\section{ARTICLES YOU MAY BE INTERESTED IN}

Electro-optical characteristics and switching behavior of the in-plane switching mode Applied Physics Letters 67, 3895 (1995); https://doi.org/10.1063/1.115309

Electro-optic characteristics and switching principle of a nematic liquid crystal cell controlled by fringe-field switching

Applied Physics Letters 73, 2881 (1998); https://doi.org/10.1063/1.122617

VOLTAGE-DEPENDENT OPTICAL ACTIVITY OF A TWISTED NEMATIC LIQUID CRYSTAL Applied Physics Letters 18, 127 (1971); https://doi.org/10.1063/1.1653593

\section{Applied Physics Letters}

Mid-IR and THz frequency combs special collection

Read Now! 


\title{
Liquid crystal display using combined fringe and in-plane electric fields
}

\author{
Ji Woong Park, ${ }^{1}$ Young Joo Ahn, ${ }^{1}$ Jun Ho Jung, ${ }^{1}$ Seung Hee Lee, ${ }^{1, a)}$ Ruibo Lu, ${ }^{2}$ \\ Hyang Yul Kim, ${ }^{2}$ and Shin-Tson $\mathrm{Wu}^{2, \mathrm{~b})}$ \\ ${ }^{1}$ Polymer BIN Fusion Research Center, Department of Polymer Nano-Science and Engineering, Chonbuk \\ National University, Chonju, Chonbuk 561-756, Republic of Korea \\ ${ }^{2}$ College of Optics and Photonics, University of Central Florida, Orlando, Florida 32816, USA
}

(Received 4 April 2008; accepted 30 July 2008; published online 25 August 2008)

\begin{abstract}
A high performance liquid crystal display using combined fringe and in-plane horizontal electric fields is proposed. The strong electric fields cause more liquid crystals to reorient almost in plane above and between the pixel electrodes. As a result, the operation voltage is lower and transmittance is higher than those of fringe field switching and in-plane switching modes, while preserving a wide viewing angle. Such a high performance device is particularly attractive for large panel liquid crystal displays. (0) 2008 American Institute of Physics. [DOI: 10.1063/1.2973152]
\end{abstract}

Large panel liquid crystal displays (LCDs) have been widely used in desktop monitors and television (TV) sets. The employed anisotropic LC functions as an independent light switch to modulate the incoming light. However, the effective LC birefringence is strongly viewing angle dependent. Thus, the image quality of the LCD is also viewing angle dependent. To overcome this viewing angle problem, many wide-view LC devices have been proposed, such as multidomain vertical alignment (MVA), ${ }^{1}$ patterned vertical alignment (PVA), ${ }^{2,3}$ in-plane switching (IPS), ${ }^{4-6}$ and fringe field switching (FFS). ${ }^{7-10}$

The MVA and PVA devices trade transmittance for wide view because they must have multiple domains tilting at different directions. The domain walls do not contribute to light modulation so that the transmittance is reduced. Moreover, the operation voltage of MVA is rather high due to the use of negative dielectric anisotropy $(\Delta \varepsilon)$ LC. On the other hand, single domain IPS exhibits a relatively wide viewing angle due to the in-plane rotation of the LC directors. However, its transmittance is low because the electric field above the pixel electrodes is too weak to reorient the LC directors. Moreover, its operation voltage is relatively high because it only uses the horizontal field. The FFS mode overcomes these problems by utilizing fringe electric fields so that transmittance occurs even above the pixel electrodes with a reduced voltage while keeping the in-plane rotation of the LC directors. However, the transmittance above the centers of pixels and common electrodes is still lower than that at the electrode edges. Thus, its overall transmittance is still lower than that of the twisted nematic mode. ${ }^{11,12}$ Its operation voltage is still high.

In LCD TVs, several lamps are used in order to produce sufficient brightness because the transmittance of LCD panel is fairly low, and frame frequency is increased from 60 to $120 \mathrm{~Hz}$ or higher in order to reduce motion picture image blurs. Many lamps and driver integrated circuits (ICs) with high output voltage and increased frequency cause heat radiation, which greatly increases the panel's temperature. This undesirable thermal effect could shift the device's electrooptic curves and exert a stress to the panel. Consequently, the

\footnotetext{
${ }^{a)}$ Electronic mail: 1sh1@ @chonbuk.ac.kr.

b)Electronic mail: swu@mail.ucf.edu.
}

image quality might be deteriorated due to increased temperature and thermal stress. ${ }^{13}$ Thus, there is an urgent need to develop a LC device with better electro-optic performances.

In this paper, we propose an improved LC device, which has higher transmittance and lower operation voltage than those of the FFS and IPS devices, while keeping a wide viewing angle. The lower operation voltage helps to reduce power consumption and heat dissipation from the driver ICs.

Figure 1 shows the electrode configuration on the bottom substrate of a FFS device and our proposed device. In the FFS device, the stripe electrodes work as signal (or pixel) electrode and plane indium tin oxide electrode works as common electrode. These two electrodes are separated by a thin passivation layer. Figure 1(a) shows the fringe electric fields of the FFS device. In the proposed device shown in Fig. 1(b), the basic structure remains the same but the pixel electrodes are not in the same potential. Instead, each alternate pixel keeps the same potential but with an opposite polarity from the adjacent pixel electrodes. Under this configuration, not only a fringe field is formed between signal and common electrodes but also an in-plane electric field is generated between signal electrodes. For the convenience of discussion, let us designate this device as fringe in-plane switching (FIS).

To explain the differences between these two devices in real driving situation, we assume that the $\mathrm{LC}$ requires $5 \mathrm{~V}$ to reach its maximum transmittance with dot inversion driving scheme. The common electrode is fixed at $5 \mathrm{~V}$ in the FFS device. The signal electrode is swung to $10 \mathrm{~V}$ in the first frame and $0 \mathrm{~V}$ in the second frame to give an ac voltage

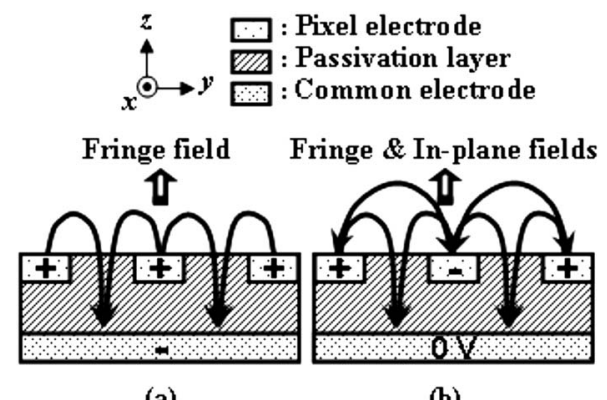

(a)

(b)

FIG. 1. Cross-sectional view of pixel structure of (a) FFS and (b) FIS devices with electric field lines. 
difference of +5 and $-5 \mathrm{~V}$, respectively. In the FFS device, the driver IC with output voltage of at least $10 \mathrm{~V}$ is required. However, in FIS, only driver IC with output voltage of $5 \mathrm{~V}$ is sufficient to drive the LC to a desired state. The common electrode is fixed at 2.5 and $5 \mathrm{~V}(0 \mathrm{~V})$ is applied to one signal and $0 \mathrm{~V}(5 \mathrm{~V})$ to the other signal electrode in the first (second) frame. Under such a circumstance, the potential difference between one (the other) signal and common electrodes is $+2.5 \mathrm{~V}(-2.5 \mathrm{~V})$; however, the potential difference between signal electrodes is +5 and $-5 \mathrm{~V}$ in the first and second frames, respectively. As a result, the equivalent operating voltage of $5 \mathrm{~V}$ to the FFS device could be realized in the FIS device using a driver IC with $5 \mathrm{~V}$ outputs, which helps to reduce driving voltage and heat dissipation.

To validate the performance advantages over the FFS and IPS devices, we simulated the electro-optic characteristics of the FIS LCD. A two dimensional LC director simulation was performed using a commercial software LCD Masters (Shintech, Japan). The optical properties were calculated using the $2 \times 2$ extended Jones matrix method. ${ }^{14,15}$ Here, the LC material with a positive $\Delta \varepsilon$ is employed with its physical properties listed as follows: $\Delta \varepsilon=8.2$, and elastic constants $K_{11}=9.7 \mathrm{pN}, K_{22}=5.2 \mathrm{pN}$, and $K_{33}=13.3 \mathrm{pN}$. The birefringence of the LC is chosen to be $\Delta n=0.10,0.09$, and 0.09 for the FFS, IPS, and FIS device to maximize their light efficiency at a given cell gap, respectively. The electrode width is $4 \mu \mathrm{m}$ and gap is $6 \mu \mathrm{m}$. The passivation layer thickness is $0.29 \mu \mathrm{m}$. The surface pretilt angle is $2^{\circ}$ with antiparallel rubbing direction between top and bottom substrates, the cell gap is $d=4 \mu \mathrm{m}$ and initial rubbing angle is $80^{\circ}$ with respect to the horizontal component of the fringe field.

When a uniaxial LC medium is sandwiched between two crossed polarizers, the normalized optical transmittance of the device is given as

$$
T / T_{o}=\sin ^{2} 2 \varphi(V) \sin ^{2} \frac{\pi d \Delta n_{\mathrm{eff}}(V)}{\lambda},
$$

where $\varphi(V)$ is voltage-dependent azimuthal component of the angle (twist angle) between the LC optic axis and the transmission axis of the polarizer, and $\Delta n_{\text {eff }}$ is effective birefringence of the LC at a given voltage $V$ and wavelength $\lambda .{ }^{16}$ In both FFS and FIS devices, the LC directors are homogenously aligned with $\varphi(V)=0$ at $V=0$. Therefore the cell appears dark at $V=0$. As the voltage exceeds a threshold, the fringe field and in-plane field jointly rotate the LC directors to generate $\varphi(V)$ and $\Delta n_{\text {eff }}$ so that the incident light transmits through the crossed analyzer.

Figure 2 shows the simulated voltage-dependent transmittance for three devices. As indicated, the operating voltage at which maximum transmittance occurs is $\sim 3.7 \mathrm{~V}_{\text {rms }}$ $\left(\sim 4.0 \mathrm{~V}_{\text {rms }}\right)$ with transmittance of $0.73(0.71)$ in the FFS (IPS) device, whereas it is $\sim 2.1 \mathrm{~V}_{\text {rms }}$ with transmittance of 0.76 in the FIS device. In other words, the operating voltage of the FIS device is only about one half of the FFS device while having a higher transmittance, owing to the higher potential difference between signal electrodes than that between signal and common electrodes.

Figures 3(a)-3(c) are the simulated LC director distributions for the FFS, IPS, and FIS modes, respectively, at their respective maximum transmittance voltages. The dark lines here are the equal potential lines from the applied electric fields. For the FFS mode shown in Fig. 3(a), the evident

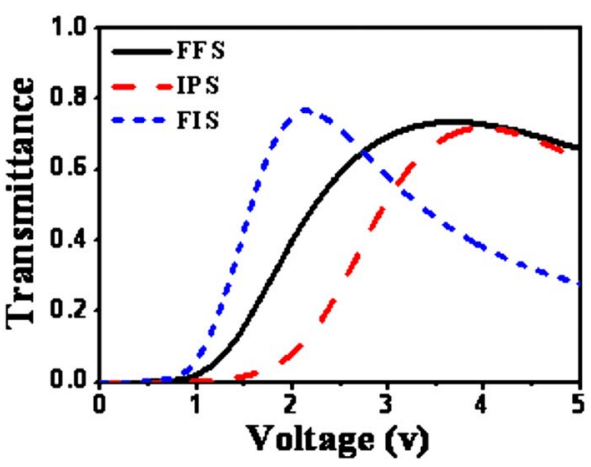

FIG. 2. (Color online) Comparison of voltage-dependent transmittance between FFS, IPS, and FIS devices.

fringe field effect exists both above the signal and the common electrodes, where the LC directors are fully switched. For the IPS device [Fig. 3(b)], the transverse electric field helps twist LC directors at the regions in between the common and the signal electrodes, where the LC directors above the electrodes are only partially tilted. For the FIS device [Fig. 3(c)], the in-plane transverse electric field exists between the two different signal electrodes above the common electrode, while the fringe field effect exists between the common electrode and the different signal electrodes.

In order to understand the transmittance difference between devices associated with field-dependent LC reorienta-

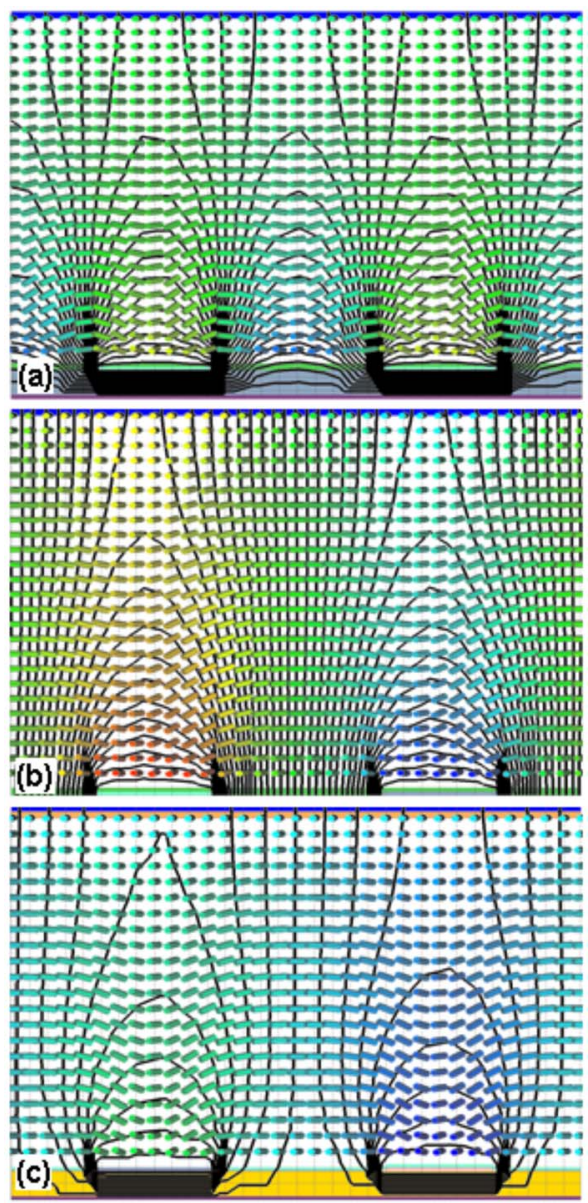

FIG. 3. (Color online) The LC director distributions of (a) FFS, (b) IPS, and (c) FIS at their respective maximum transmittance voltages. The black lines are equal potential lines. 

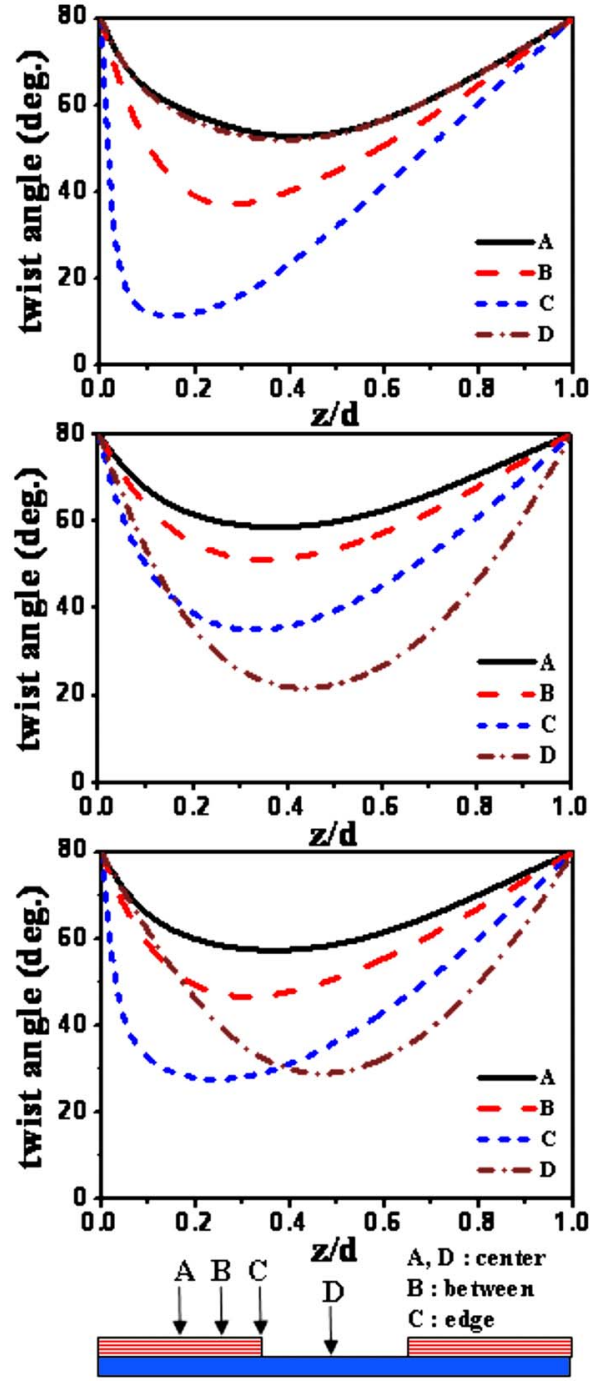

FIG. 4. (Color online) Twist angles at four different electrode positions. (a) FFS, (b) IPS, and (c) FIS.

tion, we calculated the transmittance and twist angle at various electrode positions. Results are shown in Fig. 4. In the FFS device, the transmittance is high at electrode edges but has two valleys at the center of signal and common electrodes because in these two positions $(A$ and $D)$ the LCs are twisted by the elastic torque of neighboring molecules so that the maximum twist angle is only $\sim 30^{\circ}\left(80^{\circ}-50^{\circ}\right)$, which is less than $45^{\circ}$ to lead to a maximum transmittance [see Fig. 4(a)]. In the FIS device, the horizontal as well as fringe fields between two signal electrodes are present so that the valley between electrode gap is filled giving rise to a higher transmittance. Nevertheless, the transmittance of the FIS device above signal electrode (positions $A-C$ ) is still low as compared to that of the FFS device. However, with improved transmittance between signal electrodes the overall transmittance of FIS is higher than that of FFS.
To understand why FIS exhibits a higher transmittance than IPS at above electrodes, we need to examine the fieldinduced twist angles. As indicated in Figs. 4(b) and 4(c), FIS has a smaller twist angle at positions $A$ and $B$ than FFS but few degrees higher than IPS. In addition, the average twist angle in FIS at position $C$ is much larger and closer to $45^{\circ}$ than that of IPS. In IPS, the horizontal field exists only between electrodes, but in FIS strong horizontal field exists even at electrode edges so that the LC rotates more in FIS than in IPS. This explains why FIS exhibits a higher transmittance than IPS.

Finally, the viewing angle of FIS was calculated between the full bright and dark states. The viewing cone with a contrast ratio larger than $10: 1$ is up to $80^{\circ}$, which is equivalent to those of the IPS and FFS devices. ${ }^{4,7}$ This wide viewing angle mainly originates from the in-plane rotation of the LC directors.

In conclusion, the proposed FIS device shows a much lower operation voltage and higher transmittance than the FFS mode while keeping a comparable viewing angle. The device may require two transistors and two data lines in one pixel; however, this technology is already commercialized in the LCDs using vertical alignment. We believe the proposed device is useful for the LCDs that require high transmittance, wide viewing angle, and low driving voltage.

This work was supported by the Korea Science and Engineering Foundation (Grant No. 106645) funded by the Korean government.

${ }^{1}$ A. Takeda, S. Kataoka, T. Sasaki, H. Chida, H. Tsuda, K. Ohmuro, T. Sasabayashi, Y. Koike, and K. Okamoto, SID Int. Symp. Digest Tech. Papers 29, 1077 (1998).

${ }^{2}$ K. H. Kim, K. Lee, S. B. Park, J. K. Song, S. N. Kim, and J. H. Souk, Proceedings of the 18th International Display Research Conference, 1998 (unpublished), p. 383.

${ }^{3}$ R. Lu, S. T. Wu, and S. H. Lee, Appl. Phys. Lett. 92, 051114 (2008).

${ }^{4}$ M. Oh-E and K. Kondo, Appl. Phys. Lett. 67, 3895 (1995).

${ }^{5}$ H. Hong, H. Shin, and I. Chung, J. Disp. Technol. 3, 361 (2007).

${ }^{6}$ B. S. Jung, I. S. Baik, I. S. Song, G. D. Lee, and S. H. Lee, Liq. Cryst. 33, 1077 (2006)

${ }^{7}$ S. H. Lee, S. L. Lee, and H. Y. Kim, Appl. Phys. Lett. 73, 2881 (1998).

${ }^{8}$ S. H. Lee, S. L. Lee, H. Y. Kim, and T. Y. Eom, SID Int. Symp. Digest Tech. Papers 30, 202 (1999).

${ }^{9}$ S. J. Kim, H. Y. Kim, S. H. Lee, Y. K. Lee, K. C. Park, and J. Jang, Jpn. J. Appl. Phys., Part 1 44, 6581 (2005).

${ }^{10}$ J. W. Ryu, J. Y. Lee, H. Y. Kim, J. W. Park, G. D. Lee, and S. H. Lee, Liq Cryst. 35, 407 (2008).

${ }^{11}$ M. Schadt and W. Helfrich, Appl. Phys. Lett. 18, 127 (1971).

${ }^{12}$ S. H. Lee, H. Y. Kim, S. M. Lee, S. H. Hong, J. M. Kim, J. W. Koh, J. Y. Lee, and H. S. Park, J. Soc. Inf. Disp. 10, 117 (2002).

${ }^{13}$ K. Ono, I. Mori, R. Oke, and K. Endo, Proceedings of the 14th International Display Workshops, 2007 (unpublished), p. 67.

${ }^{14}$ A. Lien, Appl. Phys. Lett. 57, 2767 (1990).

${ }^{15}$ Z. Ge, X. Zhu, T. X. Wu, and S. T. Wu, J. Opt. Soc. Am. A 22, 966 (2005).

${ }^{16}$ S. T. Wu, U. Efron, and L. D. Hess, Appl. Opt. 23, 3911 (1984). 